In this way light may be thrown upon the condition of the large intestine.

Conclusions.

1. Examinations of fæces for blood are fallacious unless previous dieting and drugs have been suitably regulated.

2. The test for soluble "albumin" in the fæces is probably even more important than that for blood (though both taken together are specially valuable); it indicates either failure of digestion and absorption of food "albumin" (a very rare condition), or the existence either of hæmorrhage or ulceration of the gut. It is almost always associated with some serious condition.

3. The presence of both "albumin" and blood in the stools indicates $(a)$ hæmorrhage with or without ulceration not very remote from the anus, or $(b)$ a severe hæmorrhage higher up the gut.

4. The presence of blood alone indicates hæmorrhage. The nearer the bleeding is to the anus, the less significance it has.

5. The presence of " albumin " alone usually indicates serous exudation, without bleeding, probably near the anus, and presumably the further the lesion from the anus, the larger the exudation must have been. More rarely it indicates a failure of digestion and to absorption of food "albumins."

6. Lavage of the large intestine, and investigation of the fluid so obtained, often gives valuable help in localising a gastro-intestinal lesion.

\section{OBSERVATIONS ON}

\section{TWO ACTIONS OF ALCOHOL}

\section{BY W. BURRIDGE, M.A., M.B., B.CH. OXF.}

THE older physicians prescribed alcohol without misgivings, and never found cause to doubt they were doing what was best for their patients. But their course of action has not hitherto been justified by experiments conducted in the laboratory, so that the modern phy sicians of necessity prescribe alcohol with an element of uncertainty. Any observations assisting them should therefore be of practical value.

Limitations of Previous Experimental Methods.

As a preliminary some possible limitations to the usual experimental method may be considered. One aim in experimental work is to obtain fixed conditions which, in the case of the heart, means perfusing that organ with some modification of Ringer's solution. The inquirer who went into any laboratory where such experiments were in progress would find the experimenter using the best possible solution for the work, and using only that solution. If he pursued his inquiries further he would find different experimenters using different "best" solutions, but each always keeping to that one he thinks best. If the action of a drug be the same under all conditions an experiment conducted under fixed conditions will give accurate and correct general results. But if the expression of the action of a drug differs under different experimental conditions, an experiment conducted under fixed conditions will give only particular results. I have found that the expression of the action of alcohol definitely differ's under different experimental conditions. This is especially the case when small amounts of the substance are used. Under the usual laboratory conditions alcohol in small amounts acts, if at all, as a depressant. But under different conditions it definitely augments cardiac activity.

We will consider first the action of 40 per cent. alcohol on the frog's heart. 'This strength is well outside the limits employed by any other experimenter, and more than one physiologist has come to me in the laboratory and expressed doubts as to the capacity of a heart to survive exposure to it. With the alcohol and the heart at hand there was no difficulty in making a convert. When I used it first I had no doubt I was using a cardiac depressant, and also that it ought to damage the heart. But certain of the facts observed did not fall directly under those headings. The heart stopped in a more or less contracted condition, and after removal of the alcohol the beats were greater in amplitude than they had been before. To bring that increase of contraction within the scope of a theory of damage or depression was difficult, but it was done. ${ }^{1}$ Such a theory, however, cannot include the additional facts following. A heart was used for experiments on the variations in the refractory period produced by this strength of alcohol. It was briefly exposed to the drug and then the disturbance so set up examined. When that disturbance had subsided the heart was briefly exposed to the alcohol again, and so a fresh disturbance set up. Thirty such disturbances were set up and the experiments lasted over two working days. On the third day the heart was still capable of activity though evidently failing. On the fourth day activity was still possible, but later ceased. The point for consideration about these experiments is that no simply perfused frog's heart has survived so long. These facts cannot be brought within the scope of a theory that the alcohol was only a depressant or that it did damage, but on the contrary. After that result one naturally turned to a re-examination of the older records showing the augmentation after the alcohol. The re-examination was greatly assisted by the discovery of a method for ascertaining the maximum contractility of the hearts on which the older experiments had been made. It was thereby found that the old experiments had been performed on hearts that were beating nearly at their maximum. They had little scope for expressing augmentation as improved contraction only, but they showed all they could. In this connexion it is as well to point out that experimenters do not in general know the capacity of the hearts on which they work. It is assumed, but not known, that the heart beats well at the start and that a deviation from that in one direction means better, and in another worse. When the maximum contraction is known it is also known whether a good or bad start was made, whether a heart is beating well or bad at a given moment and by how much, and also the activity becomes directly comparable with that of another heart.

\section{A New Iethod of Approach.}

Having thereby found that the hearts on which the original experiments were made had little scope for showing an augmentation of contraction, attempts were made to give that scope. Instead of letting the cardiac machine run approximately "all out," measures were taken to run the machine at about half power. Then an apparently different result was obtained. Instead of observing a slight increase of contraction after the heart had been treated with the alcohol a great increase was observed-as much as 100 per cent. The differences depended on possibilities. For if the machine at the start were running at 90 per cent. of its maximum, an approximately 11 per cent. increase reaches that maximum. But if at the start it were running at 50 per cent. of its maximum, a 100 per cent. increase on this reached the maximum. After treatment with 40 per cent. alcohol the heart beats at its maximum. If it were beating at 90 per cent. to begin with only 11 per cent. could thereby be added; if it were beating at 50 per cent. at the start that amplitude could be doubled, and was. In presence of the alcohol the action observed was primarily that of depression. The heart stopped and became electrically inexcitable. It stopped in a more or less contracted condition, that contracture being the only visible evidence of any augmentation at this stage. After washing away the alcohol its depression went also, and it was after this that augmentation was definitely manifest. The underlying cardiac change developed by the alcohol, and responsible for this augmentation of contraction, persisted after removal of the alcohol from the heart. It was a slowly subsiding change, and in about 20 minutes or so the heart returned to the status quo ante. This strength of alcohol, then, exerts two definite actions on the heart.

There is a depressing action coming on and going away concomitantly with the drug; there is an augmenting 
action showing a capacity for persistence after removal of its producer. The finding of that augmentation was entirely contrary to my expectations. When alcohol was first pushed in this manner it was done with a view to obtaining an advanced grade of depression. The depression was obtained, of course, but it was also found to be always accompanied by an advanced grade of augmentation. The two changes had different modes of subsidence which made possible their separation and identification. Having thus found that 40 per cent. alcohol always produced two cardiac changes, the hearts were thereafter exposed to gradually lessened strengths of the drug. Except as mentioned below, the same two actions were found. There was a depressing action coming and going concomitantly with the alcohol, there was an augmenting action showing that capacity for persistence. But on making comparison between the members of the series of experiments carried out with concentrations of alcohol varying from 0.5 per cent. to 40 per cent. there could be appreciated the existence of a definite time factor in the development of the stimulating action. The rate of development increased as the strength of alcohol was increased. With 40 per cent. alcohol a high grade of augmentation was produced in a few seconds; with 1 per cent. alcohol a lesser grade took many minutes. No such differences were observed in the rate of subsidence of the change, once it had been produced. This stimulating action of alcohol in low concentrations has been observed by W. E. Dixon ${ }^{3}$ and F. Ransom ${ }^{4}$ but its significance missed. The former though the alcohol was being metabolised, the latter that the heart was acquiring "tolerance." I do not think Dixon would suggest the phenomena observed after 40 per cent. alcohol were a result of metabolism or Ransom that they were acquired tolerance. But those phenomena are only a higher grade of the phenomena they observed and so dessribed. Neither of these observers noted the capacity for persistence of this change, nor that when it was present the cardiac refractory period was shortened.

Profound Influence of Two Factors.

The action on the heart of low concentrations of alcohol is, however, profoundly influenced by two factors not taken into account by other experimenters. The factors modifying this action are: (1) The calcium content of the perfusing solution; (2) the amplitude at which the heart is beating. When hearts beat nearly at their maximum and were perfused with a solution of relatively high calcium content-the normal laboratory conditions-small doses of alcohol ( 2 per cent. or less) either exerted no appreciable action or only depressede.g., 1 per cent. alcohol had no appreciable action, 2 per cent. slightly depressed. When hearts beat at about half their maximum and were perfused with a solution of relatively low calcium content so little as 0.5 per cent. alcohol markedly influenced cardiac activity. There was a definite though small depressing action, succeeded by an augmentation much greater than that original depression; 1 per cent. alcohol gave as much as 100 per cent. increase. Under differing experimental conditions the action of alcohol thus also differed. Those giving a positive result had obviously the greater value. Under these latter conditions the action of 0.5 per cent. alcohol differs only quantitatively from that of 40 per cent., whereas under the others there is also a qualitative difference. Calcium is chiefly respon. sible for the differences. ${ }^{2}$ This element exerts two actions on the heart. Before alcohol can depress it has to overcome the one action of calcium; and before it can augment it has to overcome the other. The more the calcium in the perfusing solution the less readily does the heart react to alcohol." Ringer's solution has a greater calcium tension than has blood, the latter containing substances such as adrenin, which enable the heart to function in presence of much less calcium than would otherwise be necessary. This greater calcium tension of Ringer's solution confers on the perfused heart a lessened capacity for change which, unless taken into consideration, can vitiate the conclusions drawn from our experiments. It can directly alter the expression of the action of a drug.
The normal heart is bathed in a solution of low calcium tension. If, in addition, it beats at.less than its maximum we have the combination of circumstances under which small amounts of alcohol exert a stimulating action far greater than their concomitant depression. Those who employed it directly under those conditions are justified. But alcohol is a substance that cannot be pushed because its depressing action increases more rapidly than the augmenting. It is only when the drug is used in small concentration that augmentation outweighs depression. Those who prescribe repeated small doses do right.

\section{Summary.}

1. Alcohol exerts two actions on the heart: $(a)$ A depressing action coming on and disappearing concomitantly with the drug; $(b)$ an augmenting action showing a variable rate of development depending on concentration, and especially a capacity for persistence after removal of its producer.

2. With increase of concentration the two actions do not increase at equal rates, depression increasing faster than augmentation.

3. Low concentrations of alcohol under the experi. mental conditions specified exert a stimulating action far greater than their concomitant depression.

\section{References.}

1. Burridge: Quart. Journ. of Exp. Physiol., p. 145, 1913.

2. Burridge: Quart. Journ. of Med., X., p. 141, 1917.

3. W. F. Dixon : Journ. of Physiol., xxxviri., 1. 346, 1907.

4. F. Ransom: Ibid., 1iii., p. 141, 1919

\section{Climital altotes:}

\section{MEDICAL, SURGICAL, OBSTETRICAL, AND THERAPEUTICAL.}

\section{A CASF ILLUSTRATING \\ RE.FORMATION OF ELBOW-JOINT \\ AFTER GUNSHOT FRACTURE AND EXTENSIVE} LOSS OF BONE.

By WinifRed F. BuCKLeY, O.B.E., M.R.C.S. ENG., LATE RESIDENT MEDICAL OFFICER, ENDELL-STREET MILITARY HOSPITAL.

I HAVE recently followed the whole history of a case in which, after gunshot injury of the left elbow-joint with fracture of the humerus and extensive loss of bone, the joint was ultimately re-formed. The notes are briefly as follows.

Private S., aged 38, was wounded on July 23rd, 1916, and admitted to the Endell-street Military Hospital on August 16th. On admission he had very septic gunsho wounds of the left elbow-joint with much swelling and œdema. The X ray plate showed a supracondylar fracture of the humerus with the lower fragment projecting obliquely forwards, its articular surface being dislocated from the head of the olecranon and radius.

On August 29th an operation was performed for the removal of the separated lower end of the humerus which was located in the joint. The inflammation did not subside for three months and there was very little movement of the joint on his discharge in December, 1916.

As he continued to attend at the out-patient pensioners department, we were able to take a series of $X$ ray plates, here reproduced, of the joint over a period of three years, and these are remarkably interesting in showing a steady growth downwards of the lower end of the humerus towards the ulna and an actual re-formation of the joint, with hardening and rounding off of the lower end of the humerus on the inner side to fit into the cavity of the ulna. The downward growth of bone continued for two vears, the new joint forming during the third year.

The photograph shows the patient's present range of movement. The left arm is two inches shorter than the right, the forearm is fixed in a position of semipronation, and there is some weakness of the muscles supplied by the musculo-spiral nerve which is improving under treatment. Otherwise good muscular power is present. 